\title{
Identify MVC architectural pattern based on ontology
}

\author{
Qiang Yin, Lulu Wang, Bixin Li \\ School of Computer Science and Engineering \\ Southeast University \\ Nanjing, China \\ \{220163720,wanglulu,bx.li\}@ seu.edu.cn
}

\begin{abstract}
MVC architectural pattern is widely used in software architecture design. It helps decouple the processing and the visualization of system data. Identified MVC architectural pattern helps understand how the software is actually implemented based on MVC architectural pattern, and further improve the consistency between design and source code. This paper proposes an ontology-based MVC architectural pattern identification method. Firstly, we use the combination of design patterns to describe the structure of MVC architectural pattern, so as to construct the MVC ontology of concept layer. Then we construct a program dependency graph by extracting the dependencies between entities in the target system, and build the ontology of instance layer. Finally, the MVC architectural pattern ontology of the specific target system is inferred by ontology reasoner in order to obtained MVC architectural pattern and the pattern elements included in each component. We use open source projects as the benchmark, and the experimental results show that our method effectively identify the MVC architectural pattern and the pattern elements in software system.
\end{abstract}

Keywords-MVC; Architectural Pattern; Pattern identification; Observer Pattern; Strategy pattern; Ontology

\section{INTRODUCTION}

Architectural style is often used to describe the architecture [1], the architectural style is also known as the software architectural pattern. MVC is a software architectural pattern that widely used in desktop applications and Web information systems ${ }^{[3]}$, which greatly improved the speed and stability of the system, also makes the software easy to maintain and easy expansion. But with the evolution of software, software design changes and the loss of software documentation make it difficult for developers to understand the architectural pattern of software. Such software is often difficult to maintain. Understanding the architectural pattern of the system is very helpful for the maintenance work. Bass et al. mention that some $80 \%$ of all costs in software development are related to maintenance activities ${ }^{[2]}$. Therefore, it is very meaningful to research the MVC software architectural pattern identification.

This paper studies the identification of MVC architectural pattern. At present, there is no set of theory and technology at home and abroad to support MVC architectural pattern identification. But there are some studies on the identification of architectural pattern. J. Paakki et al. regarded the problem that mining pattern form UML diagrams as a constraint satisfaction problem in $200{ }^{[4]}$. J. Peters proposed an architectural pattern matching method based on Semantically Rich Modular Architecture [5], using genetic algorithm to match

DOI reference number: 10.18293/SEKE2019-163 pattern instance. M. Lungu proposes an architectural pattern identification method that uses a source code structure as a pattern and builds on a lower-level pattern extracted from source code, iteratively and interactively produces a more advanced view ${ }^{[6]}$. H. Yan introduced the tool DiscoTect, which identify the architecture pattern of the runtime object-oriented system ${ }^{[7]}$. Mavridou Anastasia pointed out that architecture can be represented by logic, and architectural style can be described by configurations ${ }^{[8]}$. Thomas Haitzer proposed a semiautomated architectural pattern identification method based on architecture primitives [9]. The Service-oriented Architecture pattern identification method based on model checking was proposed by Penta and Sandonep in $2007^{[10]}$.

From the above related works, it can be concluded that the identification of architectural pattern requires two aspects of work: describing the architectural pattern and matching the description of the architectural pattern with the source code. However, the related work still has the following shortcomings.

- Lack of automation, the above methods are not highly automated, which makes it difficult to implement.

- Existing research does not identify specific pattern elements in the software system and does not provide a more detailed reference for developers and maintenance personnel. Pattern element is the pattern-related class in software system.

This paper proposes an ontology-based MVC architectural pattern identification method, which can automatically identify the MVC pattern instances in a specific target system, the pattern instances include the MVC architectural pattern and the pattern elements. The contributions of this paper mainly include the following three points. Firstly, the observer design pattern and the strategy design pattern are used to represent the MVC architectural pattern composition principle, and the ontology formally describes the MVC architectural pattern, the observer pattern and the strategy pattern. Secondly, the ontology inference engine is used to match the formal description of the MVC architectural pattern with the specific target system. The ontology inference engine can automate this process. Finally, read the source code and documentation of target system manually is used to verify the correctness of the identification result and effectiveness of the method. The recall rate, the precision and the F1-measure of the MVC architectural pattern and pattern elements are calculated to measure the effectiveness of the method.

This paper is structured as follow: In the section 2, the MVC architectural pattern and ontology are introduced. In the 


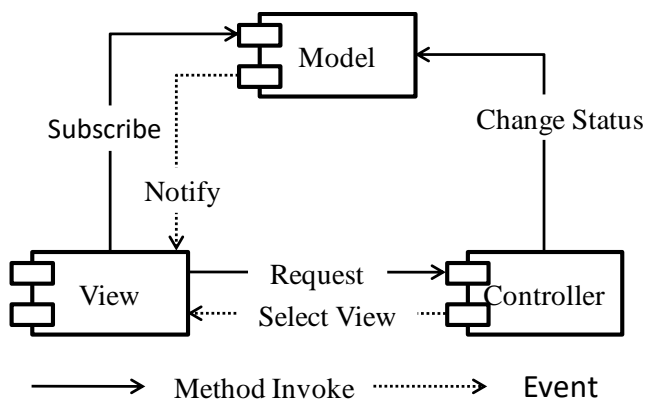

Fig. 1. MVC architectural pattern [17]

section 3 , the research method of this paper is introduced. In the section 4, the experiment and evolution is introduced. Section 5 contains conclusion and future work.

\section{BACKGROUND}

\section{A. MVC architectural pattern}

The Model-View-Controller(MVC) architectural pattern divides an interactive application into three components [11]. First created by Trygve R and implemented in the Smalltalk- 80 environment. The model contains core functions and data; the view displays information to user; the controller processes user's input. Both the view and controller form the user interface, and the change propagation mechanism ensures consistency between the user interface and the model.

MVC decouples views and models by establishing a subscribe/notify protocol between them ${ }^{[12]}$. A view must ensure that its appearance reflects the state of the model. Whenever the model's data changes, the model notifies views that depend on it. In response, each view gets an opportunity to update itself. So, the model-view relationship is an example of the Observer design pattern. The View-Controller relationship is an example of the Strategy design pattern. Encapsulate the response mechanism in the Controller object. To implement different response strategies, simply replace them with different kinds of Controller instances. Fig.1 shows MVC architectural pattern.

\section{B. Ontology}

In computer science, ontology refers to "a formal, clear and detailed description of a shared conceptual system" [13]. Ontology is used to express related concepts, entities and formal relationships between specific domains. The ontology can effectively describe the abstract relationships between specific domain concepts, and ontology can also reason in order to excavate the implicit relationship between abstract concepts. Ontologies generally use ontology language for related expression work. Currently, description logic (DL) and framework logic (FL) ${ }^{[15]}$ are generally used to define ontology. In this paper, since the description logic is used to define the ontology, the DL is introduced below.

$\mathrm{DL}$ is a formal language used for the description of ontology, the relationship between the concepts of ontology and the relationship between individuals of concepts. Description logic has excellent expressive ability and logicbased inference ability, and the description logic has clear inference algorithms ${ }^{[14]}$. The W3C international standard OWL and RDF language is based on the DL. The description logic system contains three parts: concept, role and individual. The assertion between concepts is called TBOX, and the assertion between individuals is called ABOX.

\section{1) $T B O X$}

TBOX is a set of axioms in description logic, which contains the connotation knowledge of the application domain, and uses the terminological axioms to describe the assertions between concepts. The general term axiom has two forms: inclusion and equality.

\section{2) $A B O X$}

ABOX is a set of description logic used to describe the relationship between individuals. It is an extension of the concept in the application domain to instantiate assertions axiom of individual and facts between individuals. Generally, there are two forms of instantiation axioms: concept assertion and role assertion.

The ontology of concept layer in this paper is to express through the OWL language, and the ontology of instance layer is to express through the RDF language, in order to build the ontology of MVC architectural pattern for a specific target system.

\section{MethodOLOGY}

The ontology-based MVC architectural pattern identification method proposed in this paper aims to identify the MVC architectural pattern and pattern elements in open source software. The method is mainly for the project that developed by JAVA. The method is mainly divided into three steps. Fig. 2 shows the overall process of the method.

- The first step is to represent the MVC architectural pattern as the observer pattern and the strategy pattern, and then describe the MVC architectural pattern formally by using the DL in order to form the MVC architectural pattern ontology of conceptual layer, the ontology of conceptual layer is also the Tbox in DL that mentioned in the second chapter.

- The second step is to use the code parsing tool to extract the dependencies between the entities in the source code, and use the RDF language to represent the dependencies of the entities in the source code into triples to form ontology of instance layer, the ontology of instance layer is also the Abox in DL that mentioned in the second chapter. Then, combining the MVC architectural pattern ontology of concept layer with the ontology of instance layer to form the MVC architectural pattern ontology of specific target system;

- The third step is to derive the expected result from the MVC architectural pattern ontology of the specific target system using the ontology inference engine, including whether the MVC architectural pattern is applied and the MVC architectural pattern elements in the target system. 


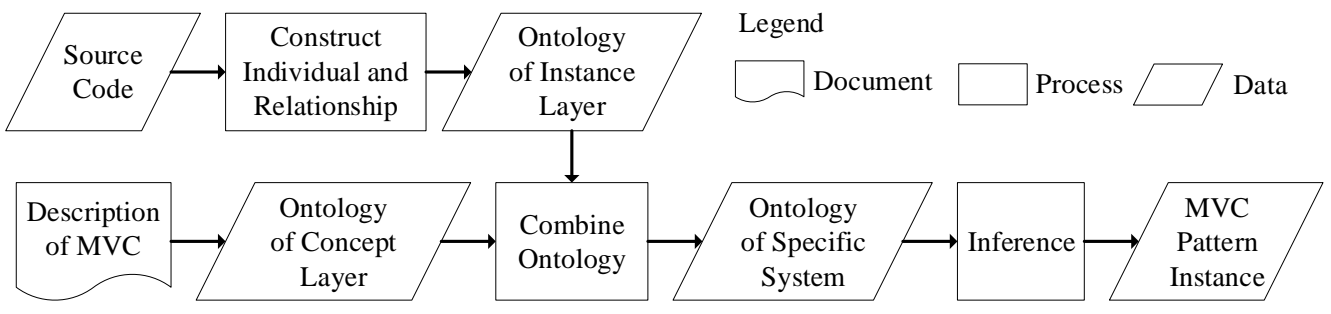

Fig. 2. The process of our method

\section{A. Ontology of concept layer}

The MVC pattern consists of three components: model, view, and controller. The pattern separates the model and view through the Observer pattern. The view is the observer in the Observer pattern. The model is the subject in the Observer pattern. Once the model data changes, the model will inform the relevant view. MVC controller is a component that accepts user input and responds to user input. The Strategy pattern is implemented, the view is the Context in the Strategy pattern, and the controller is the strategy in the Strategy pattern. On the other hand, most Java GUI applications use the Swing toolkit or the AWT toolkit, so the classes in View must inherit the base classes in JavaSwing and AWT. According to this feature, you can identify the specific class of the view in MVC.

The identification of the MVC pattern is transformed into the identification of the design pattern. The ontology of constructing the MVC architectural pattern is to construct a combination of two design pattern ontology and transform the abstract architectural pattern into a specific design pattern, thereby mapping the relationship between the pattern components to specific source code. Table I shows the DL of the main part of the ontology of concept layer.

\section{B. Ontology of specific target system}

Building the MVC architectural pattern ontology of a specific target system is mainly divided into two steps. Step one is to extract information for the target system. The purpose of information extraction is to extract the dependencies between entities in the target system in order to construct Program Dependence Graph (PDG). The entity includes methods and classes; in step two, the PDG is converted into an RDF triples, and the individual in the DL is added to the MVC architectural pattern ontology of concept layer to form an MVC architectural pattern ontology of the specific target system.

\section{1) extract information}

This paper is mainly for the Java open source project, using JAVA Development Tool(JDT) to complete the information extraction work. The main flow of JDT extraction is to first generate the Abstract Syntax Tree (AST) of the source code, and then construct the PDG according to the information on the AST.

The construction of program dependency graphs is to obtain the dependencies between classes and methods by traversing the AST. Constructing a PDG for the method of this paper requires extracting the following information, as shown in Table II.
TABLE I. DL OF THE ONTOLOGY

\section{DL of MVC pattern:}

MVCPattern $\equiv \exists$ containsElement.Model

$\cap \exists$ containsElement.View

$\cap \exists$ containsElement.Controller

\section{DL of Observer pattern:}

ObserverPattern $\equiv \exists$ containsElement.AbstractSubject

$\cap \exists$ containsElement.ConcreteSubject

$\cap \exists$ containsElement.AbstractObserver

$\cap \exists$ containsElement.ConcreteObserver

AbstractSubject $\equiv$ Interface

$\cap \exists$ containsElement. AbstractNotify

ConcreteSubject $\equiv$ NomalClass

$\cap \exists$ containsElement.ConcreteNotify

AbstractObserver $\equiv$ Interface

$\cap \exists$ containsElement.AbstractUpdate

ConcreteObserver $\equiv$ NomalClass

$\cap \exists$ containsElement.ConcreteUpdate

DL of Strategy pattern:

StrategyPattern $\equiv \exists$ containsElement.AbstractStrategy

กcontainsElement.ConcreteStrategy

กcontainsElement.Context

AbstractStrategy $\equiv$ Interface

$\cap \exists$ containsElement. AbstractAlgorithm

ConcreteStrategy $\equiv$ NomalClass

$\cap \exists$ containsElement.ConcreteAlgorithm

TABLE II. TYPE OF DEPENDENCY

\begin{tabular}{ccc}
\hline Dependency Edge & Master & Slave \\
\hline extend & Class & Class \\
implement & Class & Class \\
composite & Class & Class \\
invoke & Method & Method \\
instantiation & Method & Class \\
aggregation & Class & Class \\
\hline
\end{tabular}

\section{2) Building ontology}

Firstly, convert PDG to RDF triples, RDF (Resource Description Framework) uses the triples to represent the relationship between entities, which is divided into three parts: subject, predicate and object. It effectively describes the relationship between classes, methods and other entities in the source code, the RDF triples can be regarded as ABOX in the DL. The subject indicates that the vertex of master in the graph, the predicate indicates the dependency in the PDG, and the object indicates the vertex of slave in the PDG; then, add the RDF triples to the ontology of the MVC architectural pattern concept layer, where the subject and object of the RDF triples are regarded as the individual in the DL, the predicate of the RDF triple corresponds to the role in DL. After adding RDF triples, the MVC architectural pattern ontology of the specific target system is formed. 


\section{Inference the MVC architectural pattern}

Inference is essentially the process of drawing conclusions from existing facts according to certain rules ${ }^{[16]}$. The inference process is mainly applied to the ontology inference machine. The inference engine mainly has two functions: ontology conflict detection and acquisition of implicit knowledge. Ontology conflict detection is to ensure the logical consistency between classes and individuals in the ontology. Obtaining implicit knowledge is that inference unknown knowledge according the established rule. The current inference engines are mainly divided into two categories, one is the inference engine based on the traditional description logic. The representatives of such inference engines are RACER, FaCT++, Pellet, et al. Others the inference engine are based on inference rules. Representatives are Jena, Jess. The inference engine mainly used in this paper is the Jena inference engine.

Inferring the MVC architectural pattern ontology of specific target system is to infer the existence of the pattern instances in the ontology through the inference rule. The key step of inference is to write the reason rules. The inference rules are written according to the MVC architectural pattern composition principle. The main process is to derive the unknown facts defined in the MVC concept layer ontology based on known facts such as individual and object attributes. Individuals are classified into classes defined by the concept layer of MVC architectural pattern.

According to the MVC architectural pattern concept layer ontology, we can divide the inference rules into five parts. The first part of the inference rules is written by JavaSwing inheritance rules, the purpose is to identify the classes related to GUI. The second part of the inference rules use the composition principle of observer pattern in order to identify View and Model. The third part of the inference rule is written by the principle of strategy pattern, which is to identify View and Controller. The fourth part of the inference rule is based on the inference results of the previous three steps to infer the MVC architectural pattern and the pattern elements. The fifth part of the inference rule is common rules. As shown in Table III, it is the inference rule of the observer pattern.

TABLE III. RULES OF OBSERVER PATTERN

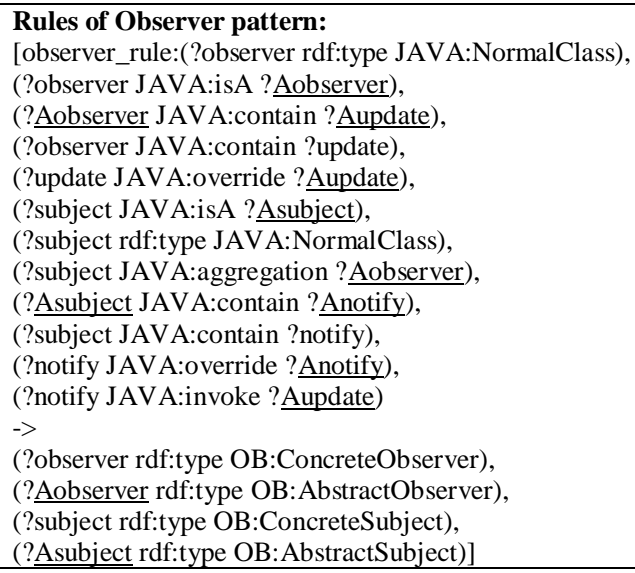

\section{EXPRIMENT AND EVALUATION}

\section{A. Purpose and metrics}

The purpose of experiment is to verify the effectiveness of our pattern identification method, we evaluate the effectiveness of our research method through three metrics: Precision, Recall, and F1-measure. Finally, analyzing the advantages and disadvantages of the method through experimental data. The calculation formula for the three metrics is as follows:

$$
\begin{array}{ll}
\text { - } & \text { Precision }=(\mathrm{TP}) /(\mathrm{TP}+\mathrm{FP}) \\
\text { - } & \text { Recall }=\mathrm{TP} /(\mathrm{TP}+\mathrm{FN}) \\
\text { F1-measure }=2 * \text { Precision } * \text { Recall / (Precision + Recall })
\end{array}
$$

\section{B. Exprimental setup}

The method of this paper is implemented in Java language. The operating system is macOS 10.12.6, the JDK version is 1.8.0_121, and the development platform is Eclipse neon.2. The MVC architectural pattern ontology is constructed by Protégé, the information extraction tool is JDT, the specific target system MVC architectural pattern ontology is constructed by Jena, and the ontology inference is completed by Jena inference engine.

\section{Result of Overrall}

In this section, we identify 20 popular open source projects, and then verify the correctness of the identification results and the effectiveness of the pattern identification method by analyzing the official documents and source code of 20 projects. Determine whether the project adopts the MVC architectural pattern by reading source code and official documentation. Finally, experimental data is obtained by comparing the recognition results with the verification results.

The results of the identification of 20 excellent open source projects are shown in the table IV. The first column of the table indicates the project name, the second column indicates the identification result, $\sqrt{ }$ indicates that the identification result is $\mathrm{MVC}, \times$ indicates that the identification result is not $\mathrm{MVC}$, and the third column indicates that actual pattern of the project, $\sqrt{ }$ indicates that the project adopts the MVC architectural pattern, and $\times$ indicates that the project does not adopt the MVC architectural pattern.

Through the results in the table $\mathrm{V}$, the statistical data is shown in table $\mathrm{V}$.

According to the calculation formula of Precision, Recall, and F1-measure, the following results can be calculated:

$$
\begin{aligned}
& \text { Precision }=(\mathrm{TP}) /(\mathrm{TP}+\mathrm{FP})=0.75 \\
& \text { Recall }=\mathrm{TP} /(\mathrm{TP}+\mathrm{FN})=1.00 \\
& \text { F1-measure }=2 * \text { Precision } * \text { Recall } /(\text { Precision }+ \text { Recall }) \\
& =0.86
\end{aligned}
$$

According to the above results, the Precision is 0.75 and the Recall is 1.00, which indicates that the method in this paper can effectively identify whether the MVC architectural 
TABLE IV. IDENTIFY RESULTS OF TWENTY PROJECTS

\begin{tabular}{ccc}
\hline Project Name & Identify Result & Validation Result \\
\hline clone & $\sqrt{ }$ & $\sqrt{ }$ \\
filezilla & $\times$ & $\sqrt{ }$ \\
freecol & $\sqrt{ }$ & $\sqrt{ }$ \\
terrier & $\sqrt{ }$ & $\times$ \\
mockito & $\times$ & $\times$ \\
lionengine & $\times$ & $\times$ \\
symphony & $\times$ & $\times$ \\
vert.x & $\times$ & $\times$ \\
okhttp & $\sqrt{ }$ & $\times$ \\
mybatis & $\times$ & $\times$ \\
jnativehook & $\times$ & $\times$ \\
jadx & $\sqrt{ }$ & $\times$ \\
jfinal-master & $\times$ & $\times$ \\
overlap2d & $\times$ & $\sqrt{ }$ \\
StormPlane & $\sqrt{ }$ & $\times$ \\
jenkins-master & $\times$ & $\times$ \\
shiro & $\times$ & $\times$ \\
pinpoint & $\times$ & $\sqrt{ }$ \\
latexdraw & $\sqrt{ }$ & \\
MARIO & $\sqrt{ }$ &
\end{tabular}

TABLE V. DATA OF IDENTIFY RESULTS

\begin{tabular}{cccc}
\hline TP & FP & TN & FN \\
\hline 6 & 2 & 11 & 0 \\
\hline
\end{tabular}

pattern is used in the software system, but there are still false positives.

\section{Result of Part}

In this section, we present three open source projects that use the MVC architectural pattern to verify the correctness of the pattern elements and the effectiveness of the method. The three open source projects are Clone, Mario, and Terrier. Firstly, it introduces the overall situation of three project. Then it gives the identification result of the three project. The identification result is the class related to the MVC pattern in the project, which is the pattern element. Then the paper understands the three projects by manually reading the source code, and classifies the classes related to MVC pattern in three projects. Finally, experimental data is obtained by comparing the identification results with the verification results.

Clone is a strategic game based on the MVC architectural pattern, which developed by Java. Mario is a popular adventure game developed in Java, similar to the Super Mario developed by Nintendo. Terrier is a highly flexible, efficient, and effective open source search engine, readily deployable on large-scale collections of documents. The basic information of the source code of three projects is shown in Table VI.

For the above three projects, due to the limitations of the paper length, we only give the identification results of Clone. The identification results of Clone are shown in Table VII.

In Table VII, classes in bold indicate false positives. The statistics of the identification results of the three projects are shown in Table VIII.

\section{E. Analysis and Conclusions}

By counting the identification results of 20 projects, we calculated that Precision is 0.75 , Recall is $1.00, \mathrm{~F} 1$-measure is 0.86 . The F1-measure is relatively high. Therefore, we can draw the conclusion that the method in this paper can effectively identify whether the MVC architectural pattern is
TABLE VI. BASIC INFORMATION ABOUT THREE PROJECTS

\begin{tabular}{cccc}
\hline Project Name & Language & Files & Code Line \\
\hline Clone & Java & 91 & 10830 \\
Mario & Java & 282 & 32859 \\
Terrier & Java & 561 & 49823 \\
\hline
\end{tabular}

TABLE VII. IDENTIFY RESULTS OF CLONE

\begin{tabular}{|c|l|}
\hline Component & \multicolumn{1}{c|}{ Pattern Element } \\
\hline \multirow{2}{*}{ Model } & $\begin{array}{l}\text { model.BoardModel; } \\
\text { boardobject.Firildak;physics.GeometryReference; } \\
\text { boardobject.Ball; physics.GeometryInterface; } \\
\text { physics.SimpleGeometry;model.Player; } \\
\text { boardobject.Cezerye; boardobject.LeftTokat; } \\
\text { boardobject.RightTokat; boardobject.Gizmo; } \\
\text { physics.GeometryCompare; } \\
\text { boardobject.Cezmi; boardobject.Observable; } \\
\text { physics.GeometryImpl; boardobject.Takoz; } \\
\text { boardobject.TriangleTakoz; boardobject.Tokat; } \\
\text { boardobject.SquareTakoz; }\end{array}$ \\
\hline $\begin{array}{l}\text { physics.GeometryImpl; observer.CezmiObserver; } \\
\text { gui.ClickHandler; gui.BeginningPanel; } \\
\text { gui.GameWindow;physics.GeometryInterface; } \\
\text { observer.GizmoObserver; observer.PlayerObserver; } \\
\text { gui.BoardPanel; gui.BuildingPanel; } \\
\text { physics.SimpleGeometry;observer.BallObserver; } \\
\text { observer.CezeryeObserver; physics.GeometryReference; } \\
\text { observer.WinPanelObserver; } \\
\text { physics.GeometryCompare; }\end{array}$ \\
$\begin{array}{l}\text { controller.DeleteGizmoController; } \\
\text { controller.AddGizmoController; } \\
\text { controller.RotateGizmoController; } \\
\text { controller.QuitController; controller.SaveController; } \\
\text { controller.PauseController; } \\
\text { controller.MoveGizmoController; } \\
\text { controller.ResumeController; controller.LoadController; } \\
\text { controller.Controller;controller.PlayController; }\end{array}$ \\
\hline
\end{tabular}

TABLE VIII. DATA OF IDENTIFY RESULTS

\begin{tabular}{ccccccc}
\hline $\begin{array}{c}\text { Project } \\
\text { Name }\end{array}$ & TP & FP & FN & Precision & Recall & F1-measure \\
\hline Clone & 39 & 7 & 0 & 0.85 & 1.00 & 0.92 \\
Mario & 128 & 54 & 21 & 0.70 & 0.86 & 0.77 \\
Terrier & 412 & 123 & 82 & 0.77 & 0.83 & 0.8 \\
\hline
\end{tabular}

used in the software system. But there are still false positives, the reasons for false positives are as follows:

There is a false positive because the method uses a rigid rule to infer whether there is an MVC architectural pattern in the software system. It does not rule out that some software systems have dependencies that satisfy the inference rules, but not the MVC architectural pattern.

By counting the identification results of three projects, we can see that the F-measure of each project is higher than 0.7 , and the value of recall is higher than precision. Therefore, we can draw the conclusion that the method of this paper can effectively identify the class related to the pattern in the software, which we call the pattern element. The main reason for the false positives is that some classes that satisfy the inference rules but are not related to the pattern are also identified. The main reason for the false negative is that some classes have the characteristics of the pattern elements, but these features are not implemented according to the standard of MVC architectural pattern. 


\section{F. Threats to validity}

There are potential threats to validity of our results. The first threat to validity is the method has only been validated for projects developed in the Java language, and has not been validated for projects developed using other object-oriented languages.

The second threat to validity is the method only validates 20 popular open source projects, and the code size of these 20 projects is less than 500k. Sample data is not very comprehensive.

\section{CONCLUSION AND FUTURE WORK}

This paper proposes an ontology-based MVC architectural pattern identification method, which can automatically identify the architectural patterns and pattern elements of MVC in software. By combining the MVC architectural pattern ontology of concept layer and the ontology of instance layer, the MVC architectural pattern ontology of the specific target system is formed, and then the ontology is inferred to obtain the MVC architectural pattern and the pattern elements. Through the statistics and analysis of experimental data, we analyzed the causes of false negatives and false positives, and summarized the advantages and disadvantages of the methods. The novelty of this method is as follows:

- Applying the design pattern to the identification of the architectural pattern makes the description of the MVC architectural pattern closer to the source code and easier to understand.

- The method also identifies the pattern elements in the software and provides developers and maintenance personnel with a more detailed reference.

- The automatic identification of the MVC architectural pattern is realized, because the method can reuse the same set of MVC architectural pattern ontology of concept layer for different systems, needn't to generate a different set of descriptions for each specific target system, and the ontology inference engine can automatically complete the architectural pattern matching process.

In the future work, firstly, we must improve the identification precision and recall of the MVC architectural pattern and pattern elements. The components and pattern elements of the MVC architectural pattern are closely related to the functionality of the software. We should consider the functional information of the class in the process of identification, so that the process is no longer limited to rigid rules; in addition, the identification of variants of the MVC architectural pattern is also worth doing, such as MVP(ModelView-Presenter) architectural pattern, which are widely used in Android, MVVM(Model-View-ViewModel) pattern using WPF technology.

\section{ACKNOWLEDGEMENT}

This work is supported in part by the National Key R\&D Program of China under Grant 2018YFB1003902, in part by the Cooperation Project with Huawei Technologies Co., Ltd., under Grant YBN2016020009, and in part by National Natural Science Foundation of China under Grant 61872078, Grant 61572126, Grant 61402103, and Grant 61572008. Special thanks to Dr. Renhao Xiong in ISEU and anonymous reviewers.

\section{REFERENCES}

[1] G. Abowd, R. Allen, D. Garlan.Using Style to Understand Descriptions of Software Architecture[C]. ACM SIGSOFT symposium on Foundations of software engineering. ACM, 1993: 9-20.

[2] L. Bass, P. Clements, R. Kazman. Software Architecture in Practice[M]. Boston, Massachusetts, USA: Addison-Wesley, 2012.

[3] CAO Shuang, Su-ling Jia. Apply MVC Architecture Pattern to C/S System[J]. Computer Knowledge and Technology, 2007-10: 946-959.

[4] J.Paakki,A.Karhinen,J.Gustafsson, etal.Softwaremetricsbyarchitecturalpa tternmining $[\mathrm{C}]$. Proceedings of the International Conference on Software: Theory and Practice. Beijing, China: Kluwer, 2000: 325-332.

[5] J. Peter, v. d. Werf. A genetic approach to architectural pattern discovery[C]. Proccedings of the European Conference on Software Architecture. Copenhagen, Denmark: ACM, 2016: 17.

[6] M. Lungu, M. Lanza, T. Girba. Package patterns for visual architecture recovery[C]. Conference on Software Maintenance \& Reengineering, Los Alamitos CA: IEEE, 2006 : 185-196.

[7] H. Yan, D. Garlan, B.Schmerl, et al. DiscoTect: a system for discovering architectures from running systems[C]. In: Proceedings of the 26th International Conference on Software Engineering. IEEE Computer Society. Washington, DC, USA: IEEE, 2004: 470-479.

[8] A. Mavridou, E. Baranov, S. Bliudze, et al. Configuration logics: Modeling architecture styles[J]. Journal of Logical and Algebraic Methods in Programming, 2017, 86(1): 2-29.

[9] T. Haitzer, U. Zdun. Semi-automatic architectural pattern identification and documentation using architectural primitives[J]. The Journal of Systems and Software, 2015, 102,: 35-57.

[10] M. D. Penta, A. Santone, M. L. Villani. Discovery of SOA Patterns via Model Checking[C]. In: 2nd International Workshop on Service Oriented Software Engineering: In Conjunction with the 6th ESEC/FSE Joint Meeting. New York, USA: ACM, 2007. 8-14.

[11] F. Buschmann, R. Meunier, H. Rohnert, et al. Pattern-Oriented Software ArchitectureVolume 1: A System of Patterns[M]. USA: Wiley, 1996.

[12] E. Gamma, R. Helm, R. Johnson, et al. Design Patterns: elements of reusable object-oriented software[M]. USA: Wiley, 1995.

[13] T. R. Gruber. A translation approach to portable ontology specifications[J]. Knowledge Acquisition - Special issue: Current issues in knowledge modeling, 1993, 5(2): 199-220.

[14] F. Baader, D. Calvanese, D. McGuinnes, et al. The Description Logic Handbook: Theory, Implementation and Applications[M]. UK, Cambridge: Cambridge University Press, 2003.

[15] L. Farinas, A. Herzig. Interference logic $=$ conditionallogic + frame axiom[J]. International Journal of Intelligent Systems, 1994, 9(1): 119130.

[16] C. Pan, H. Gu. Ontology Reasoner and Its Application[J]. Computer Systems \& Applications, 2010-09.

[17] Z. F. Ren, H. Zhang, M. S. YAN, et al. Overview of the research in model-view-controller pattern[J]. Application Research of Computers, 2004, 10: 1-4. 